\title{
Bidirectional Mendelian randomization analysis of shared genetic signals between coexisting neurodegenerative disorders to decipher underlying causal pathways
}

Sandeep Grover, International Age-related Macular Degeneration Consortium (IAMDGC)

Running head: Genetic etiology of neurodegeneration

Institut für Medizinische Biometrie und Statistik, Universität zu Lübeck, Universitätsklinikum

Schleswig-Holstein, Campus Lübeck, Lübeck, Germany

Search terms: Mendelian randomization, Causal inference, Educational attainment, Agerelated macular degeneration

Submission Type: Research article

\section{Corresponding Author:}

Sandeep Grover, Institut für Medizinische Biometrie und Statistik, Universität zu Lübeck, Ratzeburger Allee 160, 23562 Lübeck, Germany; E-mail: sandeep.grover@imbs.uni-

luebeck.de, grover.sandeep@gmail.com

Phone: +49451500 50620, +49 1732168633

Fax: +49 45150050604 


\section{ABSTRACT \\ OBJECTIVE}

To investigate whether coexistence of various neurodegenerative disorders is coincidental or biologically connected.

\section{DESIGN}

Two sample Mendelian randomization using summary effect estimates

\section{SETTING}

Genetic data taken on various neurodegenerative disorders from various cohorts comprising individuals predominantly of European ancestry.

\section{PARTICIPANTS}

International Genomics of Alzheimer's patients (IGAP), project MinE, International Agerelated Macular Degeneration Consortium (IAMDGC), International Multiple Sclerosis Genetics Consortium (IMSGC), International Parkinson's Disease Genomics Consortium (IPDGC)

\section{MAIN OUTCOME MEASURES}

Alzheimer's disease (AD), Amyotrophic lateral sclerosis (ALS), Age related macular degeneration (AMD), Multiple sclerosis (MS) and Parkinson's disease (PD).

\section{RESULTS}

A Bonferroni corrected threshold of $\mathrm{P}=0.005$ was considered to be significant, and $\mathrm{P}<0.05$ was considered suggestive of evidence for a potential association. I observed a risky effect of $\mathrm{PD}$ on $\mathrm{ALS}(\mathrm{OR}=1.126,95 \% \mathrm{CI}=1.059-1.198, \mathrm{P}=0.005)$. Using $\mathrm{AD}$ as exposure and $\mathrm{PD}$ as outcome, I observed a risky effect of $\mathrm{AD}$ on PD using all the MR methods with strongest results using $\mathrm{MBE}$ method $(\mathrm{OR}=2.072,95 \% \mathrm{CI}=1.006-4.028, \mathrm{P}=0.0416)$. Genetic predisposition to $\mathrm{AD}$ was further observed to be a risky for $\mathrm{AMD}(\mathrm{OR}=1.759,95 \% \mathrm{CI}=$ 1.040-1.974, $\mathrm{P}=0.0363)$. On the contrary, AMD was observed to be strongly protective towards $\mathrm{MS}(\mathrm{OR}=0.861,95 \% \mathrm{CI}=0.776-0.955, \mathrm{P}=0.0059)$. 


\section{CONCLUSIONS}

My findings are consistent with the previously observed relative occurrence of co-existing neurodegenerative diseases or overlapping symptoms among neurodegenerative diseases.

\section{Introduction}

It is not uncommon to see cases of neurodegeneration in clinical practice showing temporal development of one neurodegenerative disorder after another. For instance, observational studies have shown higher risk of Parkinson's disease (PD) and Alzheimers' disease (AD) among patients with a diagnosis of neovascular age-related macular degeneration (AMD) ${ }^{1-4}$. Occasional case reports of co-existence of Multiple sclerosis (MS) with Alzheimer's disease (AD) have also emerged in the literature ${ }^{5}$. Furthermore, the co-existence of Amyotrophic lateral sclerosis (ALS) with Frontotemporal dementia (FTD) had recently lead to revises consensus criteria for the diagnosis of FTD in $\mathrm{ALS}^{6}$.

Not surprisingly, most neurodegenerative diseases share certain clinical and pathological features. Furthermore, a number of genetic studies have time and again have also shown existence of shared genetic aetiology $\mathrm{y}^{7-9}$. It is common to see overlapping symptoms among various neurodegenerative disorders. For instance, up to $50 \%$ of $\mathrm{AD}$ cases exhibit aggregation of alpha-synuclein into Lewy bodies, a characteristic seen in PD cases ${ }^{10}$. Furthermore, degeneration of retinal layer, a characteristic of AMD has also been reported in cases with ALS and $\mathrm{MS}^{11,12}$. Of all the co-existing neurodegenerative, presence of ALS, parkinsonism and dementia together is the most well characterized combination often reported in specific geographic locations and known by several name such as kii-ALS or Guam-ALS or ALS$\mathrm{PDC}^{13,14}$. 
It has been long debated whether co-existence of neurodegenerative disorders is purely coincidental or there is a causal relationship in between them. However, the varying latent phases of different neurodegenerative disorders make it difficult to interpret the exact relationship between the co-existing disorders. With age as a major confounding factor in observational studies, Mendelian randomization (MR) methodology could provide an alternative solution by providing life-long effect estimates using genetic variants as proxy pseudorandomized markers of neurodegenerative diseases ${ }^{15}$. Henceforth, the objective of the current study was to explore the causal relationships among different neurodegenerative disorders using MR approach.

\section{Methods}

A two-sample MR approach was applied to explore the relationship among six most commonly occurring neurodegenerative disorders namely Alzheimer's disease (AD), Amyotrophic lateral sclerosis (ALS), Age-related macular degeneration (AMD), Frontotemporal dementia (FTD), Multiple sclerosis (MS) and Parkinson's disease (PD) ${ }^{16-20}$. I employed latest available summary GWAS datasets for the present study to prioritize genetic instruments for each of the neurodegenerative disorder. I used inverse variance weighted method as the main method to generate unconfounded estimates using the summary statistics from respective GWAS datasets to explore the relationship between each pair of neurodegenerative disorder. A Bonferroni corrected threshold of $\mathrm{P}=0.005$ (as ten pairs of neurodegernative disorders were compared) was considered to be significant, and $\mathrm{P}<0.05$ was considered suggestive of evidence for a potential association.

I also generated causal estimates adjusted for presence of potential pleiotropic variants by employing additional MR methods. The heterogeneity in the effect estimates were judged using MR-Egger, I2 and Cochrane-Q statistics. Lastly, sensitivity analysis was conducted to check reliability of estimates by excluding variants known to be directly involved in specific 
neurodegenerative disorder as an outcome, and variants believed to be potential confounders between pair of neurodegenerative disorder under consideration.

\section{Results}

All the datasets used in the study have been shown in Table 1. In addition, complete summary statistics used for causal analysis is provided in Supplementary table 1. The results from direct and reverse causal estimate analysis have been provided in Table 2a to 2e. I observed a risky effect of $\mathrm{PD}$ on $\mathrm{ALS}(\mathrm{OR}=1.126,95 \% \mathrm{CI}=1.059-1.198, \mathrm{P}=0.005)$. The risky effect was further retained using IVW, MR-Egger and WME method. Furthermore, a weak bidirectional relationship was observed between AD and PD. Using AD as exposure and PD as outcome, I observed a risky effect of $\mathrm{AD}$ on $\mathrm{PD}$ using all the MR methods with strongest results using $\mathrm{MBE}$ method $(\mathrm{OR}=2.072,95 \% \mathrm{CI}=1.006-4.028, \mathrm{P}=0.0416)$. A moderate risky effect of $\mathrm{PD}$ on $\mathrm{AD}$ was further observed using WME method $(\mathrm{OR}=1.013,95 \% \mathrm{CI}=$ 1.006-1.019, $\mathrm{P}=0.0606)$.

Genetic predisposition to AD was further observed to be a risky for AMD (OR = $1.759,95 \% \mathrm{CI}=1.040-1.974, \mathrm{P}=0.0363)$. On the contrary, AMD was observed to be strongly protective towards MS using MR-Egger method in the presence of significant pleiotropy $(\mathrm{MR}-$ Egger intercept $\mathrm{p}$-value $=0.0481, \mathrm{OR}=0.861,95 \% \mathrm{CI}=0.776-0.955, \mathrm{P}=$ $0.0059)$.

\section{Discussion}

The present study is the first study to comprehensively explore causal relationship among various neurodegenerative disorders. My results strongly confirm the genetic relationship between PD, ALS and PD as observed in the case of patients with kii-ALS or Guam-ALS or ALS-PDC. My results further suggest risky and protective effect of AMD towards $\mathrm{AD}$ and $\mathrm{MS}$ which is consistence with the relative prevalence of retinal degeneration seen in cases of AD and MS (See Supplementary table 2). 
My study has several strengths and limitations. It is one of the most comprehensive study exploiting the genetics of neurodegenerative disorders to understand the relationship among different disorders. However, differential genomic coverage and different sample sizes of different datasets make it difficult to compare the results. It is quite possible that healthy controls used in different GWAS datasets may be overlapping, leading to the risk of bias in the findings. Nevertheless, I used improved version of IVW method which takes care of these biases. Another limitation could be my inability to explore causality using different types of dementias including Frontotemporal dementia (FTD) which is known to occur in combination with ALS, as it was observed that FTD dataset was highly underpowered with sample size $<5000$ individuals.

In future, it would be important to dissect different biological pathways using relevant genetic instrument for each pair of relationships. Nevertheless, my study shows utility of genetic data to unearth important biological findings and could enhance our understanding of interconnected etiopathologies of neurodegenerative disorders. Moreover, the finding could impact the diagnosis and management of neurodegenerative disorders.

\section{Acknowledgement}

I thank Prof. Inke König for providing the institutional facilities and research environment for conduct of the research. I acknowledge the investigators of the International Age-related Macular Degeneration Consortium (IAMDGC) for sharing the summary statistics from GWAS on AMD, International Genomics of Alzheimer's patients (IGAP) on AD, International Parkinson's Disease Genomics Consortium (IPDGC) for sharing the summary statistics from GWAS on PD, project MinE for sharing the summary statistics from GWAS on ALS, and the International Multiple Sclerosis Genetics Consortium (IMSGC) for sharing the summary statistics on MS.

\section{List of figures and tables}


Table 1. Details of discovery GWAS datasets explored and prioritized instruments used for the main analysis in the present study.

Table 2a. Causal effect estimates exploring influence of various neurodegenerative disorders on Alzheimer's disaease (AD).

Table 2b. Causal effect estimates exploring influence of various neurodegenerative disorders on Amyotrophic lateral sclerosis (ALS).

Table 2c. Causal effect estimates exploring influence of various neurodegenerative disorders on Age-related macular degeneration (AMD).

Table 2d. Causal effect estimates exploring influence of various neurodegenerative disorders on Multiple sclerosis (MS).

Table 2e. Causal effect estimates exploring influence of various neurodegenerative disorders on Parkinson's disease (PD).

Supplementary table 1. Summary statistics used for the conduct of causal analysis in the current manuscript.

Supplementary table 2. Examples of recent case reports or case-series reporting co-existence of various neurodegenerative disorders (in alphabetical order).

\section{References}

1. Chung SD, Ho JD, Hu CC, Lin HC, Sheu JJ. Increased risk of Parkinson disease following a diagnosis of neovascular age-related macular degeneration: a retrospective cohort study. Am J Ophthalmol. 2014 Feb;157(2):464-9 e1.

2. Etminan M, Samii A, He B. Risk of Parkinson's disease in patients with neovascular age-related macular degeneration. J Curr Ophthalmol. 2018 Dec;30(4):365-7.

3. Seden D, Alime G, Kadir D, Serpil D, Levent T, Ozlem T. Is Alzheimer disease related to age-related macular degeneration? Turk J Med Sci. 2015;45(5):1115-21.

4. Chung SD, Lee CZ, Kao LT, Lin HC, Tsai MC, Sheu JJ. Association between neovascular age-related macular degeneration and dementia: a population-based case-control study in Taiwan. PLoS One. 2015;10(3):e0120003.

5. Luczynski P, Laule C, Hsiung GR, Moore GRW, Tremlett H. Coexistence of Multiple Sclerosis and Alzheimer's disease: A review. Mult Scler Relat Disord. 2019 Jan;27:232-8.

6. Lule DE, Aho-Ozhan HEA, Vazquez C, et al. Story of the ALS-FTD continuum retold: rather two distinct entities. J Neurol Neurosurg Psychiatry. 2019 May;90(5):586-9.

7. Ho WY, Tai YK, Chang JC, et al. The ALS-FTD-linked gene product, C9orf72, regulates neuronal morphogenesis via autophagy. Autophagy. 2019 May;15(5):827-42.

8. Clinton LK, Blurton-Jones M, Myczek K, Trojanowski JQ, LaFerla FM. Synergistic Interactions between Abeta, tau, and alpha-synuclein: acceleration of neuropathology and cognitive decline. J Neurosci. 2010 May 26;30(21):7281-9.

9. Ismail A, Cooper-Knock J, Highley JR, et al. Concurrence of multiple sclerosis and amyotrophic lateral sclerosis in patients with hexanucleotide repeat expansions of C9ORF72. J Neurol Neurosurg Psychiatry. 2013 Jan;84(1):79-87. 
10. Kotzbauer PT, Trojanowsk JQ, Lee VM. Lewy body pathology in Alzheimer's disease. J Mol Neurosci. 2001 Oct;17(2):225-32.

11. Balk $\amalg$, Steenwijk MD, Tewarie $P$, et al. Bidirectional trans-synaptic axonal degeneration in the visual pathway in multiple sclerosis. J Neurol Neurosurg Psychiatry. 2015 Apr;86(4):419-24.

12. Gabilondo I, Martinez-Lapiscina EH, Martinez-Heras E, et al. Trans-synaptic axonal degeneration in the visual pathway in multiple sclerosis. Ann Neurol. 2014 Jan;75(1):98-107. 13. Okumiya K, Wada T, Fujisawa M, et al. Amyotrophic lateral sclerosis and parkinsonism in Papua, Indonesia: 2001-2012 survey results. BMJ Open. 2014 Apr 16;4(4):e004353.

14. Plato CC, Garruto RM, Galasko D, et al. Amyotrophic lateral sclerosis and parkinsonism-dementia complex of Guam: changing incidence rates during the past 60 years. Am J Epidemiol. 2003 Jan 15;157(2):149-57.

15. Grover S, Del Greco MF, Stein CM, Ziegler A. Mendelian Randomization. Methods Mol Biol. 2017;1666:581-628.

16. Nalls MA, Blauwendraat C, Vallerga CL, et al. Expanding Parkinson's disease genetics: novel risk loci, genomic context, causal insights and heritable risk. bioRxiv. 2019 2019-01-01 00:00:00.

17. Fritsche LG, Ig| W, Bailey JN, et al. A large genome-wide association study of agerelated macular degeneration highlights contributions of rare and common variants. Nat Genet. 2016 Feb;48(2):134-43.

18. ,Patsopoulos N, Baranzini S, et al. The Multiple Sclerosis Genomic Map: Role of peripheral immune cells and resident microglia in susceptibility. bioRxiv. 2017 2017-01-01 00:00:00.

19. Jansen IE, Savage JE, Watanabe K, et al. Genome-wide meta-analysis identifies new loci and functional pathways influencing Alzheimer's disease risk. Nature Genetics. 2019 Mar;51(3):404-+.

20. van Rheenen W, Shatunov A, Dekker AM, et al. Genome-wide association analyses identify new risk variants and the genetic architecture of amyotrophic lateral sclerosis. Nat Genet. 2016 Sep;48(9):1043-8. 
Table 1. Details of discovery GWAS datasets explored and prioritized instruments used for the main analysis in the present study.

\begin{tabular}{|c|c|c|c|c|c|c|c|c|c|}
\hline S.No. & Exposure & Source study & Maximum sample size & $\begin{array}{l}\text { GWAS } \\
\text { study } \\
\text { cohort }\end{array}$ & $\begin{array}{l}\text { Number } \\
\text { of SNPs } \\
\text { analyzed }\end{array}$ & $\mathbf{P}$ & $\begin{array}{c}\begin{array}{c}\text { Number } \\
\text { of }\end{array} \\
\text { significant } \\
\text { SNPs } \\
\text { (pre- } \\
\text { clumping) }\end{array}$ & $\begin{array}{c}\begin{array}{c}\text { Number } \\
\text { of }\end{array} \\
\text { significant } \\
\text { SNPs } \\
\text { (post- } \\
\text { clumping) }\end{array}$ & $\begin{array}{c}\text { Average F- } \\
\text { statistics } \\
\text { (median } \\
\text { (range)) }\end{array}$ \\
\hline 1 & Alzheimer's disease $(\mathrm{AD})(\mathrm{AD})$ & Jansen et al. 2018 & 71880 cases/ 383,378 controls & Discovery & $133,672,99$ & $5 \times 10^{-8}$ & 2357 & 26 & $42.2(30.2-422.5)$ \\
\hline 2 & Amyotrophic lateral sclerosis (ALS) & van Rheenen et al. 2016 & 12577 cases/ 23475 controls & Discovery & $870,945,2$ & $\begin{array}{l}5 \times 10- \\
8\end{array}$ & 125 & 4 & $37.2(32.2-80.1)$ \\
\hline 3 & Age-related macular degeneration (AMD) & Fritsche et al. 2016 & 16144 cases/17832 controls & Discovery & $120,238,39$ & $5 \times 10^{-8}$ & 7218 & 42 & $47.5(29.2-382.5$ \\
\hline 4 & Multiple sclerosis (MS) (MS) & Patsopoulos et al. 2017 & 47351 cases/ 68284 controls & Discovery & $859,365,0$ & $5 \times 10^{-8}$ & 26403 & 74 & $41.9(29.8-561.9$ \\
\hline 5 & Parkinson's disease (PD) (PD) & Nalls et al. 2018 & 33,674 cases/ 449,056 controls & Discovery & $175,137,73$ & $5 \times 10^{-8}$ & 3465 & 23 & $43.6(30.0-181.5$ \\
\hline *Clum & ing was done using TwoSampleMR R packag & & & & & & & & \\
\hline
\end{tabular}


Table 2a. Causal effect estimates exploring influence of various neurodegenerative disorders on Alzheimer's disaease (AD).

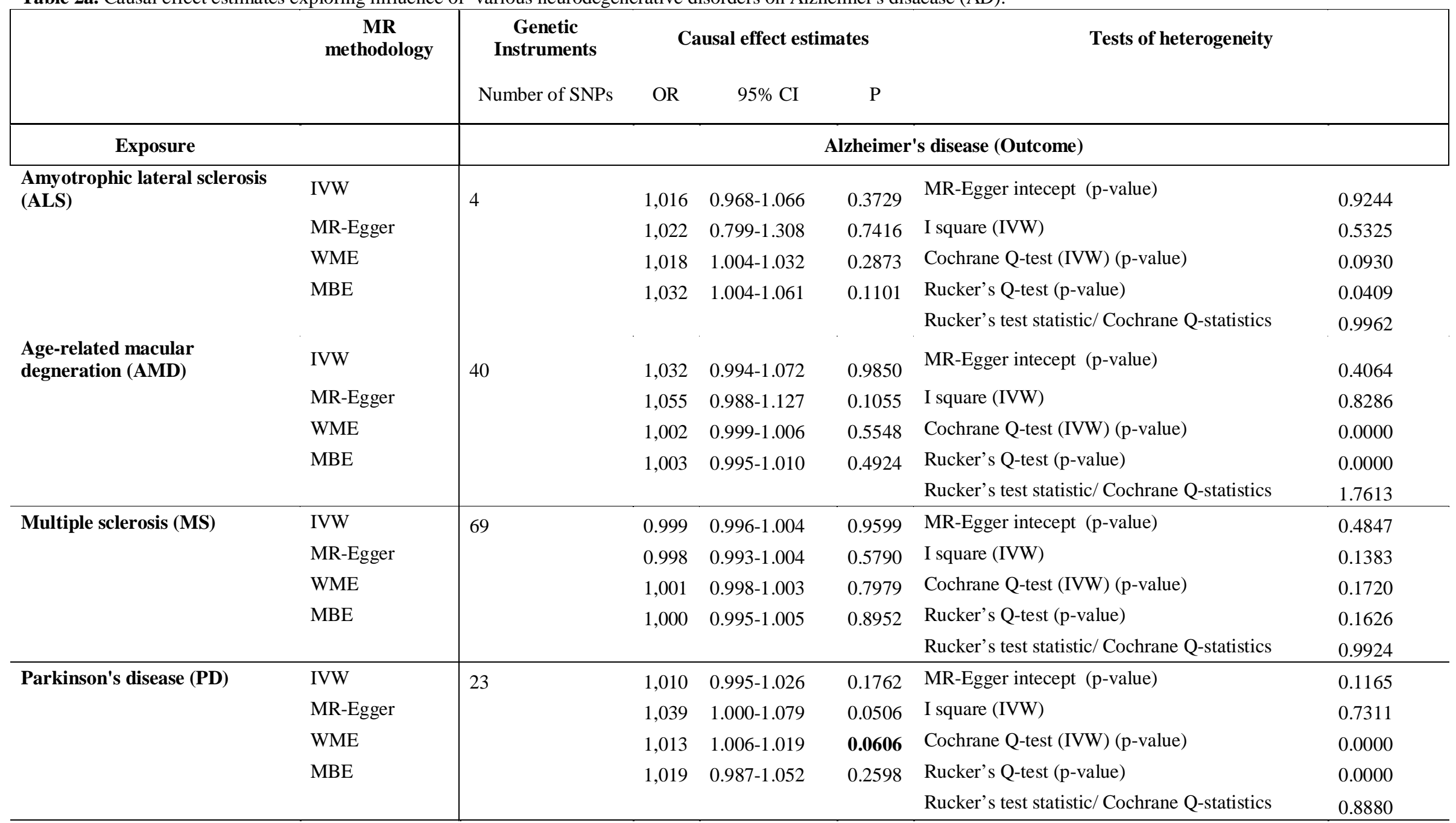


Table 2b. Causal effect estimates exploring influence of various neurodegenerative disorders on Amyotrophic lateral sclerosis (ALS).

\begin{tabular}{|c|c|c|c|c|c|c|c|}
\hline & MR & Genetic & Causa & effect estima & & Tests of heterogeneity & \\
\hline & & Number of SNPs & OR & $95 \% \mathrm{CI}$ & $\mathrm{P}$ & & \\
\hline Exposure & & Amyotrophic lat & al sclero & $\operatorname{sis}(\mathrm{ALS})(\mathrm{O} u$ & me) & & \\
\hline Alzheimer's disease (AD) & IVW & 25 & 0.996 & $0.623-1.591$ & 0.9849 & MR-Egger intecept (p-value) & 0.3992 \\
\hline & MR-Egger & & 1,729 & $0.422-7.083$ & 0.4299 & I square (IVW) & 0.0000 \\
\hline & WME & & 1,327 & $0.948-1.857$ & 0.4087 & Cochrane Q-test (IVW) (p-value) & 0.6550 \\
\hline & MBE & & 1,584 & $0.497-5.043$ & 0.4443 & Rucker's Q-test (p-value) & 0.6395 \\
\hline & & & & & & Rucker's test statistic/ Cochrane Q-statistics & 0.9670 \\
\hline $\begin{array}{l}\text { Age-related macular } \\
\text { degeneration (AMD) }\end{array}$ & IVW & 42 & 0.986 & $0.945-1.030$ & 0.5229 & MR-Egger intecept (p-value) & 0.4077 \\
\hline & MR-Egger & & 1,012 & $0.938-1.092$ & 0.7495 & I square (IVW) & 0.1823 \\
\hline & WME & & 1,002 & $0.973-1.032$ & 0.9539 & Cochrane Q-test (IVW) (p-value) & 0.1550 \\
\hline & MBE & & 1,015 & $0.948-1.086$ & 0.6724 & Rucker's Q-test (p-value) & 0.1459 \\
\hline & & & & & & Rucker's test statistic/ Cochrane Q-statistics & 0.9857 \\
\hline Multiple sclerosis (MS) & IVW & 72 & 1,008 & $0.984-1.033$ & 0.4934 & MR-Egger intecept (p-value) & 0.3323 \\
\hline & MR-Egger & & 1,020 & $0.986-1.054$ & 0.2464 & I square (IVW) & 0.0269 \\
\hline & WME & & 1,034 & $1.015-1.053$ & 0.0786 & Cochrane Q-test (IVW) (p-value) & 0.4134 \\
\hline & MBE & & 1,023 & $0.992-1.056$ & 0.1566 & Rucker's Q-test (p-value) & 0.4151 \\
\hline & & & & & & Rucker's test statistic/ Cochrane Q-statistics & \\
\hline Parkinson's disease (PD) & IVW & 22 & 1,126 & $1.059-1.198$ & 0.0006 & MR-Egger intecept (p-value) & 0.1318 \\
\hline & MR-Egger & & 1,280 & $1.068-1.533$ & 0.0099 & I square (IVW) & 0.0000 \\
\hline & WME & & 1,111 & $1.064-1.161$ & 0.0239 & Cochrane Q-test (IVW) (p-value) & 0.6965 \\
\hline & MBE & & 1,083 & $0.928-1.264$ & 0.3248 & Rucker's Q-test (p-value) & 0.7844 \\
\hline & & & & & & Rucker's test statistic/ Cochrane Q-statistics & 0.8619 \\
\hline
\end{tabular}


Table 2c. Causal effect estimates exploring influence of various neurodegenerative disorders on Age-related macular degeneration (AMD).

\begin{tabular}{|c|c|c|c|c|c|c|c|}
\hline & \multirow{2}{*}{$\begin{array}{l}\text { MR } \\
\text { methodology }\end{array}$} & \multirow{2}{*}{$\begin{array}{l}\text { Genetic } \\
\text { Instruments } \\
\text { Number of SNPs }\end{array}$} & \multicolumn{3}{|c|}{ Causal effect estimates } & \multicolumn{2}{|l|}{ Tests of heterogeneity } \\
\hline & & & OR & $95 \% \mathrm{CI}$ & $P$ & & \\
\hline \multicolumn{2}{|l|}{ Exposure } & \multicolumn{6}{|c|}{ Age-related macular degeneration (AMD) (Outcome) } \\
\hline \multirow{5}{*}{ Alzheimer's disease (AD) } & IVW & 26 & 1,759 & $1.040-2.974$ & 0.0363 & MR-Egger intecept (p-value) & 0.2093 \\
\hline & MR-Egger & & 3,150 & $1.083-9.162$ & 0.0362 & I square (IVW) & 0.3513 \\
\hline & WME & & 1,678 & $1.238-2.275$ & 0.1010 & Cochrane Q-test (IVW) (p-value) & 0.0410 \\
\hline & MBE & & 1,683 & $0.791-3.581$ & 0.1886 & Rucker's Q-test (p-value) & 0.0571 \\
\hline & & & & & & Rucker's test statistic/ Cochrane Q-statistics & 0.9295 \\
\hline \multirow[t]{5}{*}{$\begin{array}{l}\text { Amyotrophic lateral sclerosis } \\
\text { (ALS) }\end{array}$} & IVW & 4 & 0.924 & $0.679-1.256$ & 0.4717 & MR-Egger intecept (p-value) & 0.4079 \\
\hline & MR-Egger & & 1,209 & $0.369-3.959$ & 0.5629 & I square (IVW) & 0.3516 \\
\hline & WME & & 0.985 & $0.900-1.078$ & 0.8782 & Cochrane Q-test (IVW) (p-value) & 0.2013 \\
\hline & MBE & & 1,015 & $0.841-1.225$ & 0.8879 & Rucker's Q-test (p-value) & 0.2119 \\
\hline & & & & & & Rucker's test statistic/ Cochrane Q-statistics & 0.6708 \\
\hline \multirow[t]{5}{*}{ Multiple sclerosis (MS) } & IVW & 68 & 0.976 & $0.939-1.015$ & 0.2239 & MR-Egger intecept (p-value) & 0.9725 \\
\hline & MR-Egger & & 0.978 & 0.870-1.099 & 0.7054 & I square (IVW) & 0.2083 \\
\hline & WME & & 0.968 & $0.943-0.993$ & 0.2107 & Cochrane Q-test (IVW) (p-value) & 0.0717 \\
\hline & MBE & & 0.932 & $0.817-1.064$ & 0.3023 & Rucker's Q-test (p-value) & 0.0608 \\
\hline & & & & & & Rucker's test statistic/ Cochrane Q-statistics & 1.0002 \\
\hline \multirow[t]{5}{*}{ Parkinson's disease (PD) } & IVW & 23 & 0.953 & $0.901-1.007$ & 0.0863 & MR-Egger intecept (p-value) & 0.7136 \\
\hline & MR-Egger & & 0.931 & $0.806-1.074$ & 0.3098 & I square (IVW) & 0.0000 \\
\hline & WME & & 0.929 & $0.896-0.963$ & 0.0556 & Cochrane Q-test (IVW) (p-value) & 0.7723 \\
\hline & MBE & & 0.927 & $0.836-1.028$ & 0.1668 & Rucker's Q-test (p-value) & 0.7281 \\
\hline & & & & & & Cochrane Q-staitics/Rucker's test statistic & 0.9929 \\
\hline
\end{tabular}

Table 2d. Causal effect estimates exploring influence of various neurodegenerative disorders on Multiple sclerosis (MS). 


\begin{tabular}{|c|c|c|c|c|c|c|c|}
\hline & \multirow{2}{*}{$\begin{array}{l}\text { MR } \\
\text { methodology }\end{array}$} & \multirow{2}{*}{$\begin{array}{l}\text { Genetic } \\
\text { Instruments } \\
\text { Number of SNPs }\end{array}$} & \multicolumn{3}{|c|}{ Causal effect estimates } & \multicolumn{2}{|l|}{ Tests of heterogeneity } \\
\hline & & & OR & $95 \% \mathrm{CI}$ & $\mathrm{P}$ & & \\
\hline \multicolumn{2}{|l|}{ Exposure } & \multicolumn{6}{|c|}{ Multiple sclerosis (MS) (Outcome) } \\
\hline \multirow[t]{5}{*}{ Alzheimer's disease (AD) } & IVW & 26 & 1,597 & $0.257-9.908$ & 0.6020 & MR-Egger intecept (p-value) & 0.7683 \\
\hline & MR-Egger & & 3,032 & $0.025-374.146$ & 0.6388 & I square (IVW) & 0.7059 \\
\hline & WME & & 1,305 & $0.917-1.857$ & 0.4577 & Cochrane Q-test (IVW) (p-value) & 0.0000 \\
\hline & $\mathrm{MBE}$ & & 1,445 & $0.563-3.708$ & 0.4508 & Rucker's Q-test (p-value) & 0.0000 \\
\hline & & & & & & Rucker's test statistic/ Cochrane Q-statistics & 1.0094 \\
\hline \multirow[t]{5}{*}{$\begin{array}{l}\text { Amyotrophic lateral sclerosis } \\
\text { (ALS) }\end{array}$} & IVW & 3 & 0.998 & $0.629-1.582$ & 0.9860 & MR-Egger intecept (p-value) & 0.3454 \\
\hline & MR-Egger & & 1,822 & $0.016-204.153$ & 0.3528 & I square (IVW) & 0.4493 \\
\hline & WME & & 0.976 & $0.888-1.072$ & 0.8186 & Cochrane Q-test (IVW) (p-value) & 0.1627 \\
\hline & $\mathrm{MBE}$ & & 1,142 & $0.940-1.388$ & 0.3138 & Rucker's Q-test (p-value) & 0.3285 \\
\hline & & & & & & Rucker's test statistic/ Cochrane Q-statistics & 0.2629 \\
\hline \multirow[t]{5}{*}{$\begin{array}{l}\text { Age-related macular } \\
\text { degeneration (AMD) }\end{array}$} & IVW & 35 & 0.939 & 0.884-0.997 & 0.0409 & MR-Egger intecept (p-value) & 0.0481 \\
\hline & MR-Egger & & 0.861 & $0.776-0.955$ & 0.0059 & I square (IVW) & 0.4715 \\
\hline & WME & & 0.966 & $0.930-1.004$ & 0.3816 & Cochrane Q-test (IVW) (p-value) & 0.0013 \\
\hline & $\mathrm{MBE}$ & & 1,065 & $0.955-1.187$ & 0.2652 & Rucker's Q-test (p-value) & 0.0055 \\
\hline & & & & & & Cochrane Q-statistics/Rucker's test statistic & 0.8898 \\
\hline \multirow[t]{5}{*}{ Parkinson's disease (PD) } & IVW & 22 & 1,042 & $0.976-1.112$ & 0.2030 & MR-Egger intecept (p-value) & 0.5749 \\
\hline & MR-Egger & & 1,087 & $0.918-1.287$ & 0.3145 & I square (IVW) & 0.1214 \\
\hline & WME & & 1,049 & $1.005-1.095$ & 0.2722 & Cochrane Q-test (IVW) (p-value) & 0.2978 \\
\hline & $\mathrm{MBE}$ & & 1,004 & $0.863-1.167$ & 0.9627 & Rucker's Q-test (p-value) & 0.2655 \\
\hline & & & & & & Rucker's test statistic/ Cochrane Q-statistics & 0.9826 \\
\hline
\end{tabular}

Table 2e. Causal effect estimates exploring influence of various neurodegenerative disorders on Parkinson's disease (PD). 


\begin{tabular}{|c|c|c|c|c|c|c|c|}
\hline & \multirow{2}{*}{$\begin{array}{l}\text { MR } \\
\text { methodology }\end{array}$} & \multirow{2}{*}{$\begin{array}{l}\text { Genetic } \\
\text { Instruments } \\
\text { Number of SNPs }\end{array}$} & \multicolumn{3}{|c|}{ Causal effect estimates } & \multicolumn{2}{|l|}{ Tests of heterogeneity } \\
\hline & & & OR & $95 \% \mathrm{CI}$ & $\mathrm{P}$ & & \\
\hline \multicolumn{2}{|l|}{ Exposure } & \multicolumn{6}{|c|}{ Parkinson's disease (PD) (Outcome) } \\
\hline \multirow[t]{5}{*}{ Alzheimer's disease (AD) } & IVW & 26 & 2,189 & $0.996-4.812$ & 0.0510 & MR-Egger intecept (p-value) & 0.3779 \\
\hline & MR-Egger & & 1,270 & $0.289-5.584$ & 0.7418 & I square (IVW) & 0.5830 \\
\hline & WME & & 1,951 & $1.415-2.688$ & 0.0477 & Cochrane Q-test (IVW) (p-value) & 0.0001 \\
\hline & MBE & & 2,072 & $1.066-4.028$ & 0.0416 & Rucker's Q-test (p-value) & 0.0001 \\
\hline & & & & & & Rucker's test statistic/ Cochrane Q-statistics & 0.9961 \\
\hline \multirow[t]{5}{*}{$\begin{array}{l}\text { Amyotrophic lateral sclerosis } \\
\text { (ALS) }\end{array}$} & & 4 & 0.995 & $0.784-1.263$ & 0.9514 & MR-Egger intecept (p-value) & 0.8013 \\
\hline & MR-Egger & & 1,053 & $0.425-2.606$ & 0.8297 & I square (IVW) & 0.0000 \\
\hline & WME & & 0.998 & $0.915-1.089$ & 0.9844 & Cochrane Q-test (IVW) (p-value) & 0.6030 \\
\hline & MBE & & 0.952 & $0.775-1.171$ & 0.6749 & Rucker's Q-test (p-value) & 0.4118 \\
\hline & & & & & & Rucker's test statistic/ Cochrane Q-statistics & 0.9564 \\
\hline \multirow[t]{5}{*}{$\begin{array}{l}\text { Age-related macular } \\
\text { degeneration (AMD) }\end{array}$} & IVW & 38 & 0.989 & $0.945-1.035$ & 0.6427 & MR-Egger intecept (p-value) & 0.3854 \\
\hline & MR-Egger & & 0.962 & $0.887-1.042$ & 0.3300 & I square (IVW) & 0.1454 \\
\hline & WME & & 0.976 & $0.947-1.005$ & 0.4144 & Cochrane Q-test (IVW) (p-value) & 0.2205 \\
\hline & MBE & & 0.981 & $0.918-1.047$ & 0.5647 & Rucker's Q-test (p-value) & 0.2139 \\
\hline & & & & & & Rucker's test statistic/ Cochrane Q-statistics & 0.9797 \\
\hline \multirow[t]{5}{*}{ Multiple sclerosis (MS) } & IVW & 70 & 1,010 & $0.978-1.044$ & 0.5289 & MR-Egger intecept (p-value) & 0.8676 \\
\hline & MR-Egger & & 1,014 & $0.958-1.074$ & 0.6179 & I square (IVW) & 0.0776 \\
\hline & WME & & 0.984 & $0.961-1.007$ & 0.4817 & Cochrane Q-test (IVW) (p-value) & 0.2954 \\
\hline & MBE & & 0.983 & $0.932-1.038$ & 0.5438 & Rucker's Q-test (p-value) & 0.2673 \\
\hline & & & & & & Rucker's test statistic/ Cochrane Q-statistics & 0.9998 \\
\hline
\end{tabular}

